\title{
Top-quark pair hadroproduction at next-to-next-to-leading order in QCD
}

\author{
Stefano Catani, ${ }^{1}$ Simone Devoto, ${ }^{2}$ Massimiliano Grazzini, ${ }^{2}$ Stefan Kallweit, ${ }^{3}$ Javier Mazzitelli, ${ }^{2}$ and Hayk Sargsyan ${ }^{2}$ \\ ${ }^{1}$ INFN, Sezione di Firenze and Dipartimento di Fisica e Astronomia, Università di Firenze, \\ I-50019 Sesto Fiorentino, Florence, Italy \\ ${ }^{2}$ Physik Institut, Universität Zürich, CH-8057 Zürich, Switzerland \\ ${ }^{3}$ Università degli Studi di Milano-Bicocca, 20126 Milan, Italy
}

(Received 21 January 2019; published 28 March 2019)

\begin{abstract}
We report on a new calculation of the next-to-next-to-leading order QCD radiative corrections to the inclusive production of top-quark pairs at hadron colliders. The calculation is performed by using the $q_{T}$ subtraction formalism to handle and cancel infrared singular contributions at intermediate stages of the computation. We present numerical results for the total cross section in $p p$ collisions at $\sqrt{s}=8$ and $13 \mathrm{TeV}$, and we compare them with those obtained by using the publicly available numerical program ToP++. Our computation represents the first complete application of the $q_{T}$ subtraction formalism to the hadroproduction of a colorful high-mass system at next-to-next-to-leading order.
\end{abstract}

DOI: 10.1103/PhysRevD.99.051501

The top quark $(t)$ is the heaviest known elementary particle, and due to its large coupling to the Higgs boson, it is expected to play a special role in electroweak-symmetry breaking. Studies of top-quark production and decay are central in the LHC physics program, allowing us to precisely test the Standard Model and, at the same time, offering a window to possible physics beyond the Standard Model. The LHC supplies a huge number of top-quark events, thereby offering an excellent environment for such studies.

Within the Standard Model, the main source of top quarks in hadronic collisions is top-quark pair production. Studying the production of $t \bar{t}$ pairs at hadron colliders can not only shed light on the nature of the electroweaksymmetry breaking, but it also provides information on the backgrounds of many new-physics models.

Next-to-leading order (NLO) QCD corrections to the total cross section for this production process were computed 30 years ago [1-4]. The calculation of the next-tonext-to-leading order (NNLO) QCD corrections to the $t \bar{t}$ total cross section was completed a few years ago [5-8]. Besides the total cross section, differential cross sections and more general kinematical distributions are of great importance for precision studies [9]. The $t \bar{t}$ asymmetry, which is nonvanishing starting from the NLO level, is known up to NNLO [10]. Other NNLO results on differential distributions are available [11-13].

Published by the American Physical Society under the terms of the Creative Commons Attribution 4.0 International license. Further distribution of this work must maintain attribution to the author(s) and the published article's title, journal citation, and DOI. Funded by SCOAP ${ }^{3}$.
At the partonic level, the NNLO calculation of $t \bar{t}$ production requires the evaluation of tree-level contributions with two additional partons in the final state, of oneloop contributions with one additional parton, and of purely virtual contributions. The required tree-level and one-loop scattering amplitudes are known, and they are the same as those entering the NLO calculation of the associated production of a $t \bar{t}$ pair and one jet $[14,15]$. The purely virtual contributions depend on the two-loop scattering amplitudes and on the square of one-loop scattering amplitudes. Partial results for the two-loop amplitude are available in analytic form [16-19], and the complete computation has been carried out numerically $[20,21]$. The square of the oneloop amplitudes is also known [22-24].

The implementation of the above contributions in a (fully differential) NNLO calculation is a highly nontrivial task because of the presence of IR divergences at intermediate stages of the calculation. Various methods have been proposed and used to overcome these difficulties at the NNLO level (see e.g., Ref. [25] and references therein).

Partial results for $t \bar{t}$ production were obtained by using the antenna subtraction method [26,27], by considering the $q \bar{q}$ channel at leading color, and including the light-quark contributions [28-30]. The only complete NNLO computation for $t \bar{t}$ production to date is that of Refs. [5-8,10-13], which was performed by using the STRIPPER method [31-33].

In this paper, we report on a new complete computation of $t \bar{t}$ production at NNLO based on the $q_{T}$ subtraction formalism [34]. The $q_{T}$ subtraction formalism is a method to handle and cancel the IR divergences in QCD computations at NLO and NNLO accuracy. The method uses IR subtraction counterterms that are constructed by considering the transverse-momentum $\left(q_{T}\right)$ distribution of the produced 
high-mass system in the limit $q_{T} \rightarrow 0$. If the produced highmass system is composed of non-QCD (colorless) partons (e.g., leptons, vector bosons, or Higgs bosons), the behavior of the $q_{T}$ distribution in the limit $q_{T} \rightarrow 0$ has a universal (process-independent) structure that is explicitly known up to NNLO through the formalism of transverse-momentum resummation [35]. These results on transverse-momentum resummation are sufficient to fully specify the $q_{T}$ subtraction formalism for this entire class of processes. In the case of heavy-quark production, the transverse-momentum resummation formalism was developed only recently [36-38]. Nonetheless, such information was already sufficient to apply the $q_{T}$ subtraction formalism to $t \bar{t}$ production and to obtain the complete NLO corrections and the NNLO contributions in all the flavor off-diagonal channels [39]. The NNLO computation in the flavor diagonal channels requires additional perturbative information (see below), and the ensuing results are presented here for the first time.

According to the $q_{T}$ subtraction method [34], the NNLO differential cross section $d \sigma_{\mathrm{NNLO}}^{t \bar{t}}$ for the inclusive production process $p p \rightarrow t \bar{t}+X$ can be written as

$$
d \sigma_{\mathrm{NNLO}}^{t \bar{t}}=\mathcal{H}_{\mathrm{NNLO}}^{t \bar{t}} \otimes d \sigma_{\mathrm{LO}}^{i \bar{t}}+\left[d \sigma_{\mathrm{NLO}}^{i \bar{t}+\mathrm{jet}}-d \sigma_{\mathrm{NNLO}}^{t \bar{t}, C T}\right],
$$

where $d \sigma_{\mathrm{NLO}}^{\bar{t}+\text { jet }}$ is the $t \bar{t}+$ jet cross section at NLO accuracy. The square brackets term of Eq. (1) is IR finite in the limit $q_{T} \rightarrow 0$, but its individual contributions, $d \sigma_{\mathrm{NLO}}^{t \bar{t}+\mathrm{jet}}$ and $d \sigma_{\mathrm{NNLO}}^{t \bar{t}, C T}$, are separately divergent. The contribution $d \sigma_{\mathrm{NLO}}^{t \bar{t}+\text { jet }}$ can be evaluated with any available NLO method to handle and cancel IR divergences. The IR subtraction counterterm $d \sigma_{\mathrm{NNLO}}^{t \bar{t}, C T}$ is obtained from the NNLO perturbative expansion (see e.g., Refs. [39-41]) of the resummation formula of the logarithmically enhanced contributions to the $q_{T}$ distribution of the $t \bar{t}$ pair [36-38]: the explicit form of $d \sigma_{\mathrm{NNLO}}^{t \bar{t}, C T}$ is fully known.

To complete the NNLO calculation, the second-order functions $\mathcal{H}_{\mathrm{NNLO}}^{t \bar{t}}$ in Eq. (1) are needed. These functions embody process-independent and process-dependent contributions. The process-independent contributions to $\mathcal{H}_{\mathrm{NNLO}}^{i \bar{t}}$ are analogous to those entering Higgs-boson [34] and vector-boson [42] production, and they are explicitly known [43-46]. In the flavor off-diagonal channels, the process-dependent contributions to $\mathcal{H}_{\mathrm{NNLO}}^{\bar{t} \bar{t}}$ originate from the knowledge of the one-loop virtual amplitudes of the partonic processes $q \bar{q} \rightarrow t \bar{t}$ and $g g \rightarrow t \bar{t}$ and from the explicit results on the NLO azimuthal correlation terms in the transverse-momentum resummation formalism [38]. The computation of $\mathcal{H}_{\mathrm{NNLO}}^{i \bar{t}}$ in the diagonal $q \bar{q}$ and $g g$ channels additionally requires the two-loop amplitudes for $q \bar{q} \rightarrow t \bar{t}$ and $g g \rightarrow t \bar{t}$ and the evaluation of new contributions of purely soft origin. The two-loop amplitudes are available in numerical form [21], whereas the computation of the additional soft contributions has been completed by some of us [47]. ${ }^{1}$ Therefore, we are now in a position to complete the calculation of Ref. [39] and to obtain the full NNLO cross section.

Before presenting our results, we briefly describe our implementation. The NNLO cross section can be expressed as

$$
\sigma_{\mathrm{NNLO}}=\sigma_{\mathrm{NLO}}+\Delta \sigma_{\mathrm{NNLO}}
$$

The NLO contribution $\sigma_{\mathrm{NLO}}$ is evaluated by using the MuNICH code [49], which provides a fully automated implementation of the NLO dipole subtraction formalism [50-52]. We use Eq. (1) to compute the NNLO correction $\Delta \sigma_{\mathrm{NNLO}}$. The NLO cross section $d \sigma_{\mathrm{NLO}}^{\bar{t}+\mathrm{jet}}$ is computed by using MUNICH. The subtraction counterterm $d \sigma_{\mathrm{NNLO}}^{\bar{t}, C T}$ is also implemented in MUNICH, whereas the contribution proportional to $\mathcal{H}_{\mathrm{NNLO}}^{t \bar{t}}$ is evaluated with an extension of the numerical programs developed for Higgs-boson [34] and vector-boson [42] production. All the required (spin- and color-correlated) tree-level and one-loop amplitudes are obtained by using OpEnLoOPS [53], except for the fourparton tree-level color correlations that we obtain through an analytic implementation. OPENLOOPS relies on the fast and stable tensor reduction of COLLIER [54,55], supported by a rescue system based on quad-precision CutTools [56] with ONELOOP [57] to deal with exceptional phasespace points. For the purpose of validating our results for the real-virtual contribution, we have used also the new onthe-fly reduction of OPENLOOPS $2[58,59]$ and the independent matrix-element generator RECOLA [60], finding complete agreement.

The contribution in the square brackets in Eq. (1) is formally finite in the limit $q_{T} \rightarrow 0$, but both $d \sigma_{\mathrm{NLO}}^{t \bar{t}+\text { jet }}$ and $d \sigma_{\mathrm{NNLO}}^{t \bar{t}, C T}$ are separately divergent. In practice, the computation is carried out by introducing a cutoff $r_{\text {cut }}$ on the dimensionless variable $r=q_{T} / M$, where $M$ is the invariant mass of the $t \bar{t}$ pair. The final result is obtained by performing the limit $r_{\text {cut }} \rightarrow 0$. To do so, the cross section is computed at fixed values of $r_{\text {cut }}$ in the interval $\left[0.01 \%, r_{\max }\right]$. Quadratic least $\chi^{2}$ fits are performed by varying $r_{\max }$ from $0.5 \%$ to $1 \%$. The result with the lowest $\chi^{2} /$ degrees-of-freedom value is kept as the best fit. The extrapolation uncertainty is determined by comparing the result of the best fit with the results of the other fits. This procedure is the same as implemented in MATRIX [61], and it has been shown to provide a conservative estimate of the systematic uncertainty in the $q_{T}$ subtraction procedure for various processes (see Sec. 7 in Ref. [61]).

To present our quantitative results, we consider $p p$ collisions at $\sqrt{s}=8$ and $13 \mathrm{TeV}$, and we use the NNPDF31 [62] NNLO parton distribution functions

\footnotetext{
${ }^{1}$ An independent computation of these soft contributions was recently presented in Ref. [48].
} 
TABLE I. Total cross section for $t \bar{t}$ production in $p p$ collisions. The quoted uncertainties are obtained through scale variations as described in the text. Numerical uncertainties on the last digit are stated in parentheses (and include the $r_{\text {cut }} \rightarrow 0$ extrapolation uncertainties).

\begin{tabular}{lcc}
\hline \hline$\sigma_{\mathrm{NNLO}}(\mathrm{pb})$ & $q_{T}$ subtraction & TOP++ \\
\hline $8 \mathrm{TeV}$ & $238.5(2)_{-6.3 \%}^{+3.9 \%}$ & $238.6_{-6.3 \%}^{+4.0 \%}$ \\
$13 \mathrm{TeV}$ & $793.4(6)_{-5.7 \%}^{+3.5 \%}$ & $794.0_{-5.7 \%}^{+3.5 \%}$ \\
\hline \hline
\end{tabular}

throughout. The QCD running of $\alpha_{\mathrm{S}}$ is evaluated at threeloop order with $\alpha_{\mathrm{S}}\left(m_{Z}\right)=0.118$, and the pole mass of the top quark is fixed to $m_{t}=173.3 \mathrm{GeV}$. The central values of the renormalization $\left(\mu_{R}\right)$ and factorization $\left(\mu_{F}\right)$ scales are fixed to $\mu_{R}=\mu_{F}=m_{t}$. We start the presentation by considering the complete NNLO cross sections. In Table I, our results at $\sqrt{s}=8$ and $13 \mathrm{TeV}$ are compared with the corresponding results obtained with the numerical program ToP++ [63], ${ }^{2}$ which implements the NNLO calculation of Refs. [5-8] (at NLO ToP++ uses the parametrization of Refs. [64,65] of the analytic result of Ref. [66]). In Table I, the NNLO cross sections are reported with their perturbative uncertainty, which is estimated through the customary procedure of independently varying $\mu_{R}$ and $\mu_{F}$ by a factor of 2 around their central value with the constraint $0.5 \leq \mu_{F} / \mu_{R} \leq 2$. The program ToP++ gives results without an associated numerical error. Our results are given with an uncertainty that is obtained by combining statistical errors from the Monte Carlo integration and the systematic uncertainty associated to the $r_{\text {cut }} \rightarrow 0$ extrapolation. Such combined uncertainty turns out to be at the per mille level, and our results are consistent with those of ToP++ for all the considered values of $\mu_{R}$ and $\mu_{F}$.

In Table II, the NNLO corrections $\Delta \sigma_{\mathrm{NNLO}}$ in the various partonic channels $a b \rightarrow t \bar{t}+X$ computed with $q_{T}$ subtraction are compared to the corresponding results obtained with ToP++. The contribution from all the channels with $a b=q g, \bar{q} g$ is labeled as $q g$, and the contribution from all the channels with $a b=q q, \bar{q} \bar{q}, q q^{\prime}, \bar{q} \bar{q}^{\prime}, q \bar{q}^{\prime}, \bar{q} q^{\prime}$ is labeled as $q(\bar{q}) q^{\prime}$.

We see that the numerical uncertainties of our NNLO corrections are at the percent level or smaller, except for the $q g$ contribution at $\sqrt{s}=13 \mathrm{TeV}$, for which there is a large cancellation between the two terms in Eq. (1) (the term that is proportional to $\mathcal{H}_{\mathrm{NNLO}}^{\bar{t}}$ and the term in the square brackets). Similar effects were already observed in Ref. [39]. Comparing our $8 \mathrm{TeV}$ results for $\Delta \sigma_{\mathrm{NNLO}}$ with those obtained by using TOP++, we see that they are fully compatible within $1 \sigma$. At $13 \mathrm{TeV}$, we also find agreement at the $1 \sigma$ level apart from the $q \bar{q}$ channel that exhibits a $1.6 \sigma$ difference, which corresponds to about $0.5 \%$ of $\Delta \sigma_{\mathrm{NNLO}}$ in this

\footnotetext{
${ }^{2}$ The program ToP++ is used with the input parameter Precision $=3$.
}

TABLE II. NNLO corrections $\Delta \sigma_{\mathrm{NNLO}}$, split into the different production channels, for $\mu_{R}=\mu_{F}=m_{t}$. Numerical integration errors on the last digits are stated in parentheses.

\begin{tabular}{lccccc}
\hline \hline & \multicolumn{2}{c}{$q_{T}$ subtraction } & & \multicolumn{2}{c}{ ToP++ } \\
\cline { 2 - 3 }$\Delta \sigma_{\mathrm{NNLO}}(\mathrm{pb})$ & $8 \mathrm{TeV}$ & $13 \mathrm{TeV}$ & & $8 \mathrm{TeV}$ & $13 \mathrm{TeV}$ \\
\hline$g g$ & $25.77(23)$ & $80.99(54)$ & & 25.86 & 81.54 \\
$q \bar{q}$ & $2.249(12)$ & $4.713(16)$ & & 2.248 & 4.739 \\
$q g$ & $-2.349(31)$ & $-4.16(19)$ & & -2.340 & -4.089 \\
$q(\bar{q}) q^{\prime}$ & $0.1563(11)$ & $0.6378(34)$ & & 0.1563 & 0.6375 \\
\hline \hline
\end{tabular}

channel. Considering the partly statistical nature of our error estimate and the fact that the uncertainties from ToP++ were completely neglected in this discussion, we can state that our results are in agreement with the ToP++ results throughout.

The quality of the $r_{\text {cut }} \rightarrow 0$ extrapolation can be assessed by investigating the behavior of the cross section at fixed values of $r_{\text {cut }}$. In Fig. 1, we study this behavior in the different partonic channels. We see that the $r_{\text {cut }}$ dependence is larger than what is observed in the case of the production of a colorless system (see Sec. 7 of Ref. [61]), where the powerlike dependence of the total cross section on $r_{\text {cut }}$ is known to be quadratic (modulo logarithmic enhancements). In the case of $t \bar{t}$ production, due to the additional contribution of soft radiation from the heavy quarks, the $r_{\text {cut }}$ dependence of the cross section is expected to be linear [67], thereby implying a stronger sensitivity to the parameter $r_{\text {cut }}$. The exception is the $q(\bar{q}) q^{\prime}$ channel, where the expected $r_{\text {cut }}$ dependence should be quadratic, since this channel does not receive contributions from soft radiation at NNLO.

To conclude, we have reported on a new complete computation of the $t \bar{t}$ cross section in hadron collisions at NNLO in QCD perturbation theory. The computation is performed by combining tree-level and one-loop QCD amplitudes, as obtained from OPENLOOPS, with two-loop contributions available from the literature. The results are obtained by using the $q_{T}$ subtraction formalism to handle and cancel IR singularities. The contributions needed to apply $q_{T}$ subtraction to this process that were previously unknown have been computed by some of us, and they will be reported in a separate publication. We have presented numerical results in $p p$ collisions at 8 and $13 \mathrm{TeV}$ and compared them to the corresponding results obtained with the numerical program ToP++. We find good agreement within the numerical uncertainties. Our computation represents the first complete application of the $q_{T}$ subtraction formalism to the hadroproduction of a colorful high-mass system at NNLO. The computation can be naturally extended to differential distributions and by applying arbitrary IR safe cuts on the $t \bar{t}$ pair and the associated QCD radiation. More details on the calculation and additional results will be presented elsewhere. 

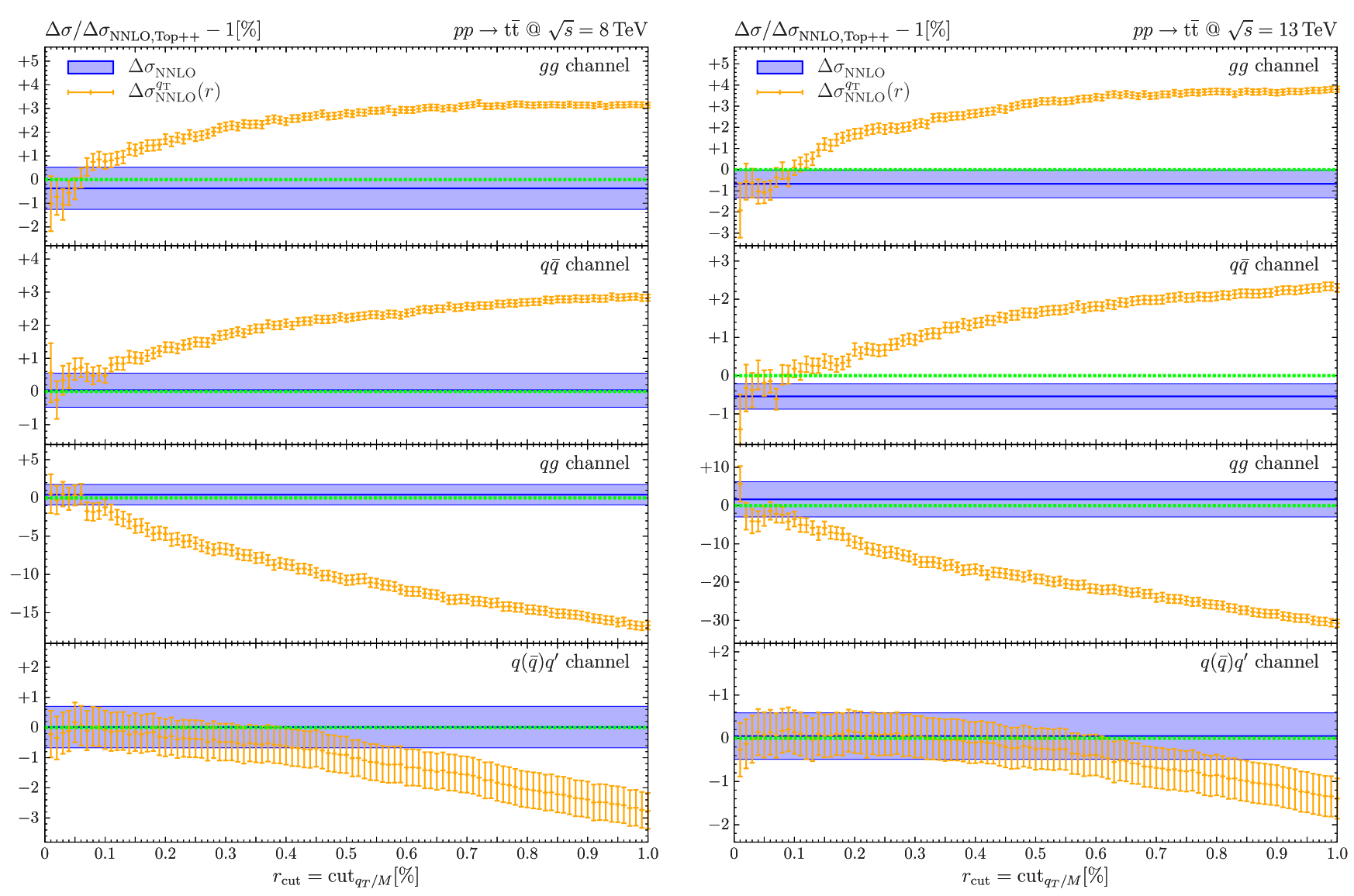

FIG. 1. NNLO corrections $\Delta \sigma_{\mathrm{NNLO}}$ normalized to the ToP++ result as a function of $r_{\text {cut }}$ in the various channels. The (blue) bands represent our final extrapolated result with its uncertainty.

\section{ACKNOWLEDGMENTS}

We are most grateful to Federico Buccioni, Jean-Nicolas Lang, Jonas Lindert, Stefano Pozzorini, and Max Zoller for their continuous assistance on issues related to OPENLOOPS during the course of this project and for allowing us to use a preliminary version of OPENLOOPS 2. This work is supported in part by the Swiss National Science Foundation (SNF) under Contract No. 200020-169041. The work of S. K. is supported by the ERC Starting Grant No. 714788 REINVENT.
[1] P. Nason, S. Dawson, and R. K. Ellis, Nucl. Phys. B303, 607 (1988).

[2] W. Beenakker, H. Kuijf, W. L. van Neerven, and J. Smith, Phys. Rev. D 40, 54 (1989).

[3] W. Beenakker, W. L. van Neerven, R. Meng, G. A. Schuler, and J. Smith, Nucl. Phys. B351, 507 (1991).

[4] P. Nason, S. Dawson, and R. K. Ellis, Nucl. Phys. B327, 49 (1989); B335, 260(E) (1990).

[5] P. Bärnreuther, M. Czakon, and A. Mitov, Phys. Rev. Lett. 109, 132001 (2012).

[6] M. Czakon and A. Mitov, J. High Energy Phys. 12 (2012) 054.

[7] M. Czakon and A. Mitov, J. High Energy Phys. 01 (2013) 080 .
[8] M. Czakon, P. Fiedler, and A. Mitov, Phys. Rev. Lett. 110, 252004 (2013).

[9] M. L. Mangano, P. Nason, and G. Ridolfi, Nucl. Phys. B373, 295 (1992).

[10] M. Czakon, P. Fiedler, and A. Mitov, Phys. Rev. Lett. 115, 052001 (2015).

[11] M. Czakon, D. Heymes, and A. Mitov, Phys. Rev. Lett. 116, 082003 (2016).

[12] M. Czakon, P. Fiedler, D. Heymes, and A. Mitov, J. High Energy Phys. 05 (2016) 034.

[13] M. Czakon, D. Heymes, and A. Mitov, arXiv:1704.08551.

[14] S. Dittmaier, P. Uwer, and S. Weinzierl, Phys. Rev. Lett. 98, 262002 (2007). 
[15] S. Dittmaier, P. Uwer, and S. Weinzierl, Eur. Phys. J. C 59, 625 (2009).

[16] R. Bonciani, A. Ferroglia, T. Gehrmann, D. Maitre, and C. Studerus, J. High Energy Phys. 07 (2008) 129.

[17] R. Bonciani, A. Ferroglia, T. Gehrmann, and C. Studerus, J. High Energy Phys. 08 (2009) 067.

[18] R. Bonciani, A. Ferroglia, T. Gehrmann, A. von Manteuffel, and C. Studerus, J. High Energy Phys. 01 (2011) 102.

[19] R. Bonciani, A. Ferroglia, T. Gehrmann, A. von Manteuffel, and C. Studerus, J. High Energy Phys. 12 (2013) 038.

[20] M. Czakon, Phys. Lett. B 664, 307 (2008).

[21] P. Bärnreuther, M. Czakon, and P. Fiedler, J. High Energy Phys. 02 (2014) 078.

[22] J. G. Korner, Z. Merebashvili, and M. Rogal, Phys. Rev. D 77, 094011 (2008); 85, 119904(E) (2012).

[23] C. Anastasiou and S. M. Aybat, Phys. Rev. D 78, 114006 (2008).

[24] B. Kniehl, Z. Merebashvili, J. G. Korner, and M. Rogal, Phys. Rev. D 78, 094013 (2008).

[25] J. R. Andersen et al., arXiv:1803.07977.

[26] A. Gehrmann-De Ridder, T. Gehrmann, and E. W. N. Glover, J. High Energy Phys. 09 (2005) 056.

[27] G. Abelof and A. Gehrmann-De Ridder, J. High Energy Phys. 04 (2011) 063.

[28] G. Abelof and A. Gehrmann-De Ridder, J. High Energy Phys. 12 (2014) 076.

[29] G. Abelof, A. Gehrmann-De Ridder, and I. Majer, J. High Energy Phys. 12 (2015) 074.

[30] G. Abelof, A. Gehrmann-De Ridder, P. Maierhöfer, and S. Pozzorini, J. High Energy Phys. 08 (2014) 035.

[31] M. Czakon, Phys. Lett. B 693, 259 (2010).

[32] M. Czakon, Nucl. Phys. B849, 250 (2011).

[33] M. Czakon and D. Heymes, Nucl. Phys. B890, 152 (2015).

[34] S. Catani and M. Grazzini, Phys. Rev. Lett. 98, 222002 (2007).

[35] S. Catani, L. Cieri, D. de Florian, G. Ferrera, and M. Grazzini, Nucl. Phys. B881, 414 (2014).

[36] H. X. Zhu, C. S. Li, H. T. Li, D. Y. Shao, and L. L. Yang, Phys. Rev. Lett. 110, 082001 (2013).

[37] H. T. Li, C. S. Li, D. Y. Shao, L. L. Yang, and H. X. Zhu, Phys. Rev. D 88, 074004 (2013).

[38] S. Catani, M. Grazzini, and A. Torre, Nucl. Phys. B890, 518 (2015).

[39] R. Bonciani, S. Catani, M. Grazzini, H. Sargsyan, and A. Torre, Eur. Phys. J. C 75, 581 (2015).

[40] G. Bozzi, S. Catani, D. de Florian, and M. Grazzini, Nucl. Phys. B737, 73 (2006).

[41] G. Bozzi, S. Catani, D. de Florian, and M. Grazzini, Nucl. Phys. B791, 1 (2008).
[42] S. Catani, L. Cieri, G. Ferrera, D. de Florian, and M. Grazzini, Phys. Rev. Lett. 103, 082001 (2009).

[43] S. Catani and M. Grazzini, Eur. Phys. J. C 72, 2013 (2012); 72, 2132(E) (2012).

[44] S. Catani, L. Cieri, D. de Florian, G. Ferrera, and M. Grazzini, Eur. Phys. J. C 72, 2195 (2012).

[45] T. Gehrmann, T. Lübbert, and L. L. Yang, Phys. Rev. Lett. 109, 242003 (2012).

[46] T. Gehrmann, T. Lübbert, and L. L. Yang, J. High Energy Phys. 06 (2014) 155.

[47] S. Catani, S. Devoto, M. Grazzini, and J. Mazzitelli (to be published).

[48] R. Angeles-Martinez, M. Czakon, and S. Sapeta, J. High Energy Phys. 10 (2018) 201.

[49] S. Kallweit (to be published).

[50] S. Catani and M. H. Seymour, Phys. Lett. B 378, 287 (1996).

[51] S. Catani and M.H. Seymour, Nucl. Phys. B485, 291 (1997); B510, 503(E) (1998).

[52] S. Catani, S. Dittmaier, M. H. Seymour, and Z. Trocsanyi, Nucl. Phys. B627, 189 (2002).

[53] F. Cascioli, P. Maierhöfer, and S. Pozzorini, Phys. Rev. Lett. 108, 111601 (2012).

[54] A. Denner, S. Dittmaier, and L. Hofer, Proc. Sci., LL2014 (2014) 071.

[55] A. Denner, S. Dittmaier, and L. Hofer, Comput. Phys. Commun. 212, 220 (2017).

[56] G. Ossola, C. G. Papadopoulos, and R. Pittau, J. High Energy Phys. 03 (2008) 042.

[57] A. van Hameren, Comput. Phys. Commun. 182, 2427 (2011).

[58] F. Buccioni, S. Pozzorini, and M. Zoller, Eur. Phys. J. C 78, 70 (2018).

[59] F. Buccioni, J. N. Lang, J. Lindert, P. Maierhöfer, S. Pozzorini, H. Zhang, and M. Zoller (to be published).

[60] A. Denner, J. N. Lang, and S. Uccirati, Comput. Phys. Commun. 224, 346 (2018).

[61] M. Grazzini, S. Kallweit, and M. Wiesemann, Eur. Phys. J. C 78, 537 (2018).

[62] R. D. Ball et al. (NNPDF Collaboration), Eur. Phys. J. C 77, 663 (2017).

[63] M. Czakon and A. Mitov, Comput. Phys. Commun. 185, 2930 (2014).

[64] U. Langenfeld, S. Moch, and P. Uwer, Phys. Rev. D 80, 054009 (2009).

[65] M. Aliev, H. Lacker, U. Langenfeld, S. Moch, P. Uwer, and M. Wiedermann, Comput. Phys. Commun. 182, 1034 (2011).

[66] M. Czakon and A. Mitov, Nucl. Phys. B824, 111 (2010).

[67] S. Catani, M. Grazzini, and H. Sargsyan, J. High Energy Phys. 06 (2017) 017. 